\title{
ON APPROXIMATION IN BA SPACES FOR JACKSON-MATSUOKA POLYNOMIALS ON THE SPHERE
}

\author{
GuO FEng And YuAN Feng
}

Abstract. We consider the best approximation by Jackson-Matsuoka polynomials on the unit sphere of $\mathbb{R}^{d}$ in the $B a$ space. Establish and use the relations between $K$-functionals and modulus of smoothness on the sphere, we obtain the direct and inverse estimate of approximation by these polynomials for the $h$-spherical harmonics.

Mathematics subject classification (2010): 41A25, 41A35, 41A63.

Keywords and phrases: Jackson-Matsuoka polynomials, best approximation, Ba spaces, modulus of smoothness, $K$-functionals, $h$-spherical harmonics.

\section{REFERENCES}

[1] X. X. Ding, P. Z. Luo, Ba spaces and some estimates of Laplace operator, J.Sys. Sci. and Math. Sci, 1981, 1: 9-33.

[2] A. Erdlyi, W. Magnus, F. Oberhettinger, and F. G. Tricomi, Higher Transcendental Functions, Vols. 1, 2, 3, McGraw-hill, New York, NY, USA, 1953.

[3] YUAN XU, Approximation by means of $h$-harmonic polynomials on the unit sphere, Adv. Comput. Math., 2004, 21: 37-58.

[4] Y. Matsuoka, On the approximation of functions by some singular integrals, Tohoku Math. J., 1966, 18: $13-43$.

[5] E. Belinsky, F. Dai, Z. Ditzian, Multivariate approximating averages, J Approx. Theory, 2003, 125: 85-105.

[6] W. Z. Chen, Approximation Theory of Operators, Xiamen Univ. Publishing House, 1989, (In Chinese).

[7] G. FEng, Y. FEng, Direct and inverse strong-type inequalities for Jackson-matsulka polnomials on the sohere, Journal of mathematical Inequalities, 2013, 7 (1): 33-41.

[8] G. R. Chen, T. Y. Zhao, Some Functional Spaces and Problems, Journal of Baji College of Arts and Sciences (Natural Science), 1997, 17 (4): 14-18, (In Chinese). 